\title{
Recurrence rates and factors affecting recurrence after traumatic anterior shoulder dislocation
}

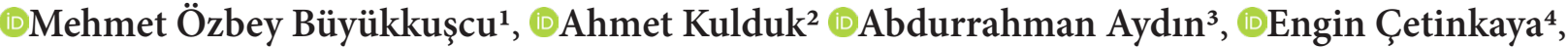 \\ @Ş̧ükrü Sarper Gürsu ${ }^{3}$ \\ ${ }^{1}$ Health Science University Gaziosmanpaşa Training and Research Hospital, Department of Orthopaedics and Traumatology, İstanbul, Turkey \\ ${ }^{2}$ Fenerbahçe Beko Basketball Team, Orthopaedics and Traumatology, İstanbul, Turkey \\ ${ }^{3}$ Health Science University Baltalimanı Bone Diseases Education and Research Hospital, Department of Orthopaedics and Traumatology, \\ İstanbul, Turkey \\ ${ }^{4}$ Health Science University İstanbul Başakşehir Pine and Sakura City Training and Research Hospital, Department of Orthopaedics and \\ Traumatology, İstanbul, Turkey
}

Cite this article as: Büyükkuşcu MÖ, Kulduk A, Aydın A, Çetinkaya E, Gürsu ŞS. Recurrence rates and factors affecting recurrence after traumatic anterior shoulder dislocation. Anatolian Curr Med J 2021; 3(2); 140-144.

\begin{abstract}
Objective: To investigate the factors affecting the recurrence of anterior shoulder dislocation, identify the patient group with the highest recurrence rates of shoulder dislocation, and determine the patient population to be recommended surgical treatment after the primary dislocation.

Materials and Method: We retrospectively screened the patients diagnosed with a primary shoulder dislocation and treated in our hospital between January 2005 and January 2017. Of the 1395 patients identified, we reached 1253 over phone calls to obtain follow-up information. The study excluded patients with no follow-up information, those with posterior dislocations, multidirectional instabilities, general joint hypermobility, traumatic nerve injury or shoulder fracture-dislocations (including greater tuberosity and glenoid fractures), and surgical treatment after a primary dislocation. We evaluated the general joint laxity of the patients using the Beighton scale. We recruited patients with a traumatic primary anterior shoulder dislocation and at least two years of follow-up data and divided them into three age groups: under 20 years, 20 to 40 years, and over 40 years.

Results: After applying the exclusion and inclusion criteria, 1,004 patients were included in the study. We detected recurrence in 408 patients (40.6\%). The group under 20 years had the highest recurrence rate with 52\% (88/170). We determined the recurrence rates as 43\% (283/659) in the group aged $20-40$ years and $21 \%(37 / 175)$ in the group over 40 years, respectively. However, except for age, we reached gender, presence of bone defects (Hill-Sachs lesion), and remaining parameters (immobilization time, injury mechanism, exercise habits and type of sports activity, dominant-side dislocation, and smoking) had no significant impact on the risk of recurrence after a primary dislocation.

Conclusion: The surgical treatment option should be presented to young male patients with bone defects after a primary dislocation. They also need to be informed about the possible difficulties due to recurrent instability.
\end{abstract}

Keywords: Shoulder dislocation, first, recurrence, risk factors, surgical treatment

\section{INTRODUCTION}

Shoulder joint dislocation is the most common joint dislocation encountered in emergency departments. Although its incidence reported in the literature varies depending on the population, this rate is estimated to be between 11-51/100,000 (1-5). Shoulder are more prone to redislocation following the first dislocation. In the literature, the rate of redislocation is generally reported as $17 \%-96 \%(6,7)$. The most consistent and significant factor affecting the prognosis of shoulder dislocation is the age of the patient at the time of the primary dislocation (8-10). Although still controversial, prognostic factors shown to affect recurrent instability also include low socioeconomic status, glenoid and/or humeral bone loss, exercise habits and type of sports, gender, and occupation $(9,11,12)$.

This study aimed to investigate the factors affecting the recurrence of shoulder dislocation, identify the group of patients with the highest recurrence rates of shoulder dislocation, and determine the population to be recommended surgical treatment after the primary dislocation. We hypothesized that young people and those with bone defects would have higher rates of redislocation. 


\section{MATERIALS AND METHOD}

Before initiating the study procedures, we obtained the relevant approval from the Medical Specialty Ethics Committee of Health Science University Baltalimani Bone Diseases Education and Research Hospital (dated 01.19.2019, no. 22). Moreover, we conducted the study in accordance with the principles of the Declaration of Helsinki.

We retrospectively screened patients who presented to the emergency department of Baltalimani Bone Diseases Education and Research Hospital with an isolated primary shoulder dislocation between January 2005 and January 2017 and whose treatments and follow-ups were performed at this hospital. We were able to gather the information of 1253 of the 1395 patients via the electronic patient registration system and over phone calls. The study included patients with traumatic primary anterior shoulder dislocations and at least two years of follow-up. Nevertheless, we excluded 55 patients with unavailable patient information, 9 cases accompanied by nerve damages, 49 with posterior dislocations, 7 with multidimensional instabilities, 27 with general joint hypermobility, and 102 with shoulder fracturedislocations (including bony Bankart lesions and greater tuberosity fractures). Considering the inclusion and exclusion criteria, we conducted the study with a total of 1,004 patients.

The retrospectively-reviewed data revealed all the procedures performed on the patients at the hospital. The patients were taken for vascular nerve examination after diagnosing shoulder joint dislocation in the emergency department based on physical examination and radiological imaging (anteroposterior and transthoracic shoulder joint radiography) findings. Then, closed reduction (Milch or Kocher Method) was applied, and immobilization was achieved with a shoulder arm sling. The vascular nerve examination and radiological imaging were repeated to check the state after reduction. In all patients, the shoulder joint was monitored at internal rotation at $0^{\circ}$ abduction for one-three or four-six weeks with a shoulder arm sling. Outpatient controls were performed at the third and sixth weeks and third, sixth and twelfth months.

We evaluated the general joint laxity of the patients using the Beighton scale, accepting a score of $\geq 4$ as general joint hypermobility (13). Besides, we extracted the anamnesis and physical examination findings of the patients during their visits to the emergency department and outpatient clinics from the patient registration system. We obtained the information which was not available in the patient registry system, (the mechanism of injury-causing primary dislocation, history of injury, smoking, exercise habits, presence of recurrent instability, and time and cause of redislocation and reason) over phone interviews. We utilized computed tomography to evaluate the shoulder joints of some patients (suspected fractures or bone defect measurements). Overall, we evaluated the shoulders of all patients with the help of magnetic resonance (MR) imaging at the sixth-week outpatient control.

In line with the previous research, we divided the patients into three age groups: under 20 years, 20 to 40 years, and over 40 years $(5,11)$. We investigated the relationship between the likelihood of recurrence and patient age, gender, smoking, cause of primary dislocation, presence of a humerus bone defect (Hill-Sachs lesion), exercise habits, and effect of dominant-side dislocation.

\section{Rehabilitation after Immobilization}

The patients were allowed to move their elbows, wrists, and hand joints during shoulder fixation. Then, the patients were included in a physiotherapy program. The rehabilitation program covered scapular stabilization exercises and aimed to strengthen the rotator cuff and deltoid muscle. The patients started recommended exercises after the sixth week to ensure proprioceptive control. The patients were allowed to resume their sports activities after three months.

\section{Statistical Analysis}

We used Statistical Product and Service Solutions (SPSS) v. 22.0 for the statistical analyses. We presented the descriptive data as mean, standard deviation, median, maximum, minimum, frequency, and percentage. We run a Chi-square test to analyze independent qualitative data. A p-value of $<0.05$ was considered statistically significant.

\section{RESULTS}

Table 1 shows the demographic characteristics and smoking status of the patients, recurrence rates and times, and the presence of bone defects. While the lowest patient age was 16 years, the highest was 64 years. After the primary dislocation, we could not detect recurrence in 596 (59.4\%) of 1,004 shoulders. However, we determined more than one dislocation in $408(40.6 \%)$ patients. Also, we identified that $62 \%$ $(n=262)$ of the shoulders with more than one dislocation underwent surgery due to recurrent instability, but the remaining 161 patients were not operated on despite recurrent dislocation. The group aged under 20 years had the highest recurrence rate with $52 \%$. We determined the recurrence rate as $43 \%$ in the group aged $20-40$ years and $21 \%$ in the one aged over 40 years, respectively. There was no significant difference between the patients under 20 years and those aged 20-40 years by the rate of recurrent dislocations. However, these rates were significantly 
higher in the groups aged under 20 years and 20 to 40 years compared to those aged over 40 years. Another striking finding was that the rate of recurrent dislocations was significantly higher in males than in females. The patients with bone defects were also more likely to have recurrent dislocation (Table 2). Finally, we found that immobilization time, injury mechanism, exercise habits and type of sports activity, dominant-side dislocation, and smoking had no significant impact on the recurrence risk after a primary dislocation (Table 2).

\begin{tabular}{|c|c|c|c|}
\hline & & $\mathbf{n}$ & $\%$ \\
\hline \multirow{3}{*}{ Age } & $<20$ & 170 & $17.0 \%$ \\
\hline & $20-40$ & 659 & $65.8 \%$ \\
\hline & $>40$ & 175 & $17.2 \%$ \\
\hline \multirow{2}{*}{ Gender } & Female & 220 & $22.0 \%$ \\
\hline & Male & 784 & $78.0 \%$ \\
\hline \multirow{2}{*}{ Smoking } & $(-)$ & 613 & $61.0 \%$ \\
\hline & $(+)$ & 391 & $39.0 \%$ \\
\hline \multirow{2}{*}{$\begin{array}{l}\text { Dominant-side } \\
\text { dislocation }\end{array}$} & $(-)$ & 425 & $42.0 \%$ \\
\hline & $(+)$ & 579 & $58.0 \%$ \\
\hline \multirow{3}{*}{ Sports activity } & None & 643 & $65.0 \%$ \\
\hline & Contact sports & 256 & $25.0 \%$ \\
\hline & $\begin{array}{l}\text { Non-contact sports/ } \\
\text { Over-head sports }\end{array}$ & 105 & $10.0 \%$ \\
\hline \multirow{2}{*}{$\begin{array}{l}\text { Immobilization } \\
\text { time }\end{array}$} & One-three weeks & 697 & $69.4 \%$ \\
\hline & Four-six weeks & 307 & $30.6 \%$ \\
\hline \multirow{4}{*}{$\begin{array}{l}\text { Injury } \\
\text { mechanism }\end{array}$} & Falls & 454 & $45.3 \%$ \\
\hline & Sports injuries & 341 & $34.0 \%$ \\
\hline & Fight, battery & 162 & $16.0 \%$ \\
\hline & Motorbike accident & 47 & $4.7 \%$ \\
\hline \multirow{2}{*}{ Hill-Sachs lesion } & $(-)$ & 321 & $32.1 \%$ \\
\hline & $(+)$ & 683 & $68.2 \%$ \\
\hline \multirow{2}{*}{ Recurrence } & $(-)$ & 596 & $59.4 \%$ \\
\hline & $(+)$ & 408 & $40.6 \%$ \\
\hline
\end{tabular}

\section{DISCUSSION}

In the literature, recurrence rate of traumatic primary anterior shoulder dislocations varies between 17\% and $96 \%$ (14-16). In the current study, we found the recurrence rate of traumatic primary anterior shoulder dislocations, for which non-operative treatment had been applied, to be $40.6 \%$. We also determined that young male patients with bone defects were at higher risk of recurrent instability compared to the general population. However, the remaining factors had no significant effect on the risk of recurrence.

In all current case series studies, the leading risk factor for recurrence is shown to be patient age at the time of primary dislocation (17-19). Lill et al. (20), investigating recurrent dislocation rates in 45 patients under 30 years and 46 patients over 30 years, found these rates to be $89 \%$ $(n=40)$ in the former group and $26 \%(n=12$ patients) in the latter group. The difference between the two groups was statistically significant. In a 25 -year follow-up of patients with primary traumatic anterior shoulder dislocation, Hovelius et al. (21) reported that $39 \%$ of the patients aged $17-19$ years (15 of 38 patients), 33\% of the patients aged 20 25 years (19 of 57 patients), $26 \%$ of the patients aged $26-29$ years (7 of 27 patients) and $14 \%$ of the patients aged $30-40$ years (9 of 63 patients) had received operative treatment due to recurrent instability at the time of primary traumatic shoulder dislocation. In the current study, consistent with the literature, we found the recurrence rates to be significantly higher in patients aged under 20 years and 20 to 40 years compared to those over 40 years.

\begin{tabular}{|llcccccc}
\multicolumn{7}{l}{ Table 2. Prognosis by demographic characteristics, smoking, immobilization time, sports activity, bone defect, and injury mechanism. } \\
\hline
\end{tabular}


In a systematic review that defined prognostic factors for recurrent instability, Wasserstein et al. (1) reported that none of the factors showed a significant relationship with recurrent instability, except for age. Shields et al. (11) stated that gender was not a risk factor for redislocation or recurrent instability. In addition to research indicating that gender does not affect recurrent instability, the literature bears many studies suggesting that male gender is an important prognostic factor $(5,9,16,21,22)$. In a study releasing the 25-year long-term outcomes of 257 primary anterior shoulder dislocation cases, Hovelius et al. (23) reported no significant difference between females and males by recurrence rates. Similarly, we concluded recurrence rates of primary traumatic anterior shoulder dislocation to be significantly higher in males than in females.

In the long-term follow-up study of Hovelius (21), the primary anterior shoulder dislocation cases under 40 years of age were divided into three groups as those doing contact sports, those doing other sports, and those not engaged in any sports. As a result, there was no significant difference between the groups by recurrent dislocation rates. Both teSlaa (9) and Simonet (24) investigated the effect of engaging in sports on the risk of recurrent dislocation. Neither study reported a significant relationship between before the primary dislocation and recurrent dislocation by exercise habits. In the current study, we divided the patients into three groups as those engaged in contact sports, those engaged in non-contact sports, and those not performing any sports activities. As a result, we determined that neither the type of sports performed before primary dislocation nor exercise habits had any significant effect on recurrent dislocation.

Much of thinking in the literature concentrates on the effect of immobilization duration on recurrence. It was previously concluded that it did not alter the risk of recurrent dislocations $(20,21,25,26)$. In the present study, we divided the patients into two groups (one-three weeks and four-six weeks) by the immobilization period. Consequently, the groups did not differ significantly by recurrent dislocation rates.

We did not encounter a study examining the relationship between smoking and recurrent dislocation in nonoperatively treated traumatic primary anterior shoulder dislocation cases. In our study, we determined no significant relationship between smoking and redislocation among the patients.

Hovelius (27) reported that the injury mechanism causing primary traumatic anterior shoulder dislocation had no significant impact on recurrent dislocation. Sachs (28) and Robinson (5) also noted that the injury mechanism did not influence recurrence rates in conservatively treated traumatic shoulder dislocation cases. In our study, we accepted the injury mechanism as a fall even in patients that fell off a 2-meter height during sports activities. Other injury mechanisms were identified as sports injuries, fight-battery, motorbike accident, and seizure. Consistent with the literature, we also found no significant relationship between the injury mechanism and redislocation risk.

Kralinger (6), Hovelius (27), and Olds (29) reported higher recurrence rates in the presence of Hill-Sachs lesion after a primary anterior dislocation. Both Henry (30) and Safran (31) reported no significant difference in redislocation rates between those with and without Hill-Sachs lesions after a primary anterior shoulder dislocation. Also, Salomonsson (32) and Hoelen (33) found no significant difference between those with and without Hill Sachs lesions by the likelihood of recurrent instability. In the current study, we discovered that the presence of Hill-Sachs lesions having developed during the primary anterior dislocation had a significant impact on recurrence. In accordance with the relevant literature, dominant-side dislocation also did not have any impact on recurrence (33-35).

There are some limitations of this study. The first is concerned with the retrospective design and the absence of an extended follow-up. Another limitation is that the patients did not have any clinical scoring. Lastly, since we conducted the study retrospectively in a single-center, it was not a fully epidemiological study. Despite these limitations, it was the strength of the research that it included clinical and radiological examinations, as well as a large number of patients.

\section{CONCLUSION}

The surgical treatment option should be presented to young male patients with bone defects after a primary dislocation, and they need to be informed about the possible difficulties due to recurrent instability.

\section{ETHICAL DECLARATIONS}

Ethics Committee Approval: The study was carried out with the permission of Medical Specialty Ethics Committee of Health Science University Baltalimani Bone Diseases Education and Research Hospital (dated 01.19.2019, no. 22).

Informed Consent: Because the study was designed retrospectively, no written informed consent form was obtained from patients..

Referee Evaluation Process: Externally peer-reviewed.

Conflict of Interest Statement: The authors have no conflicts of interest to declare. 
Financial Disclosure: The authors declare that this study received no financial support.

Author Contributions: All of the authors declare that they all participated in the design, execution, and analysis of the paper and that they approved its final version.

\section{REFERENCES}

1. Wasserstein DN, Sheth U, Colbenson K, et.al. The true recurrence rate and factors predicting recurrent instability after nonsurgical management of traumatic primary anterior shoulder dislocation: a systematic review. Arthroscopy 2016; 32: 2616-25.

2. Boffano M, Mortera S, Piana R. Management of the first episode of traumatic shoulder dislocation. EFORT Open Rev 2017; 13: 35-40.

3. Kane P, Bifano SM, Dodson CC, Freedman KB. Approach to the treatment of primary anterior shoulder dislocation: A review. Phys Sportsmed 2015; 43: 54-64.

4. Khiami F, Gérometta A, Loriaut P. Management of recent firsttime anterior shoulder dislocations. Orthop Traumatol Surg Res 2015; 101: 51-7.

5. Robinson CM, Howes J, Murdoch H, Will E, Graham C. Functional outcome and risk of recurrent instability after primary traumatic anterior shoulder dislocation in young patients. J Bone Joint Surg Am 2006; 88: 2326-36.

6. Kralinger FS, Golser K, Wischatta R, Wambacher M, Sperner G. Predicting recurrence after primary anterior shoulder dislocation. Am J Sports Med 2002; 30: 116-20.

7. Pehlivan O. Open surgical stabilization of anterior glenohumeral instability: Indications and early term results. Eklem Hastalik Cerrahisi 2002; 13: 136-40.

8. Sofu H, Gürsu S, Koçkara N, Oner A, Issın A, Camurcu Y Recurrent anterior shoulder instability: Review of the literature and current concepts. World J Clin Cases 2014; 16: 676-82.

9. teSlaa RL, Wijffels MP, Brand R, Marti RK. The prognosis following acute primary glenohumeral dislocation. J Bone Joint Surg Br 2004; 86: 58-64.

10. Kavaja L, Lähdeoja T, Malmivaara A, Paavola M. Treatment after traumatic shoulder dislocation: a systematic review with a network meta-analysis. Br J Sports Med 2018; 52: 1498-506.

11. Shields DW, Jefferies JG, Brooksbank AJ, Millar N, Jenkins PJ. Epidemiology of glenohumeral dislocation and subsequent instability in an urban population. J Shoulder Elbow Surg 2018; 27: 189-95.

12. Olds MK, Ellis R, Parmar P, Kersten P. Who will redislocate his/ her shoulder? Predicting recurrent instability following a first traumatic anterior shoulder dislocation. BMJ Open Sport Exerc Med 2019; 7: 5 .

13. Juul-Kristensen B, Rogind $\mathrm{H}$, Jensen DV, Remvig L Interexaminer reproducibility of tests and criteria for generalized joint hypermobility and benign joint hypermobility syndrome. Rheumatology (Oxford) 2007; 46: 1835-41.

14. Marshall T, Vega J, Siqueira M, Cagle R, Gelber JD, Saluan P. Outcomes after arthroscopic bankart repair: patients with firsttime versus recurrent dislocations. Am J Sports Med 2017; 45: 1776-82.

15. van Kampen DA, van den Berg T, van der Woude HJ, Castelein RM, Terwee CB, Willems WJ. Diagnostic value of patient characteristics, history, and six clinical tests for traumatic anterior shoulder instability. J Shoulder Elbow Surg 2013; 22: 1310-9.

16. Vermeiren J, Handelberg F, Casteleyn PP, Opdecam P. The rate of recurrence of traumatic anterior dislocation of the shoulder: a study of 154 cases and a review of the literature. Int Orthop 1993; 17: $337-41$.
17. Jakobsen BW, Johannsen HV, Suder P, Søjbjerg JO. Primary repair versus conser-vative treatment of first-time traumatic anterior dislocation of the shoulder: a randomized study with 10year follow-up. Arthrosc J Arthrosc Relat Surg 2007; 23: 118-23.

18. Scheibel M, Kuke A, Nikulka C, Magosch P, Ziesler O, Schroeder RJ. How longshould acute anterior dislocations of the shoulder be immobilized in externalrotation? Am J Sports Med 2009; 37: 1309-16.

19. Smith TO. Immobilisation following traumatic anterior glenohumeral joint dis-location: a literature review. Injury 2006 37: 228-37.

20. Lill H, Korner J, Hepp P, Verheyden P, Josten C. Age-dependent prognosis following conservative treatment of traumatic anterior shoulder dislocation. Eur J Trauma 2001; 27: 29-33.

21. Hovelius L, Olofsson A, Sandström B, et al. Non-operative treatment of primary anterior shoulder dislocation in patients forty years of age and younger. a prospective twenty-five-year follow-up. J Bone Joint Surg Am 2008; 90: 945-52.

22. Leroux T, Wasserstein D, Veillette C, et al. Epidemiology of primary anterior shoulder dislocation requiring closed reduction in Ontario, Canada. Am J Sports Med 2014; 42: 442-450.

23. Hovelius L, Rahme H. Primary anterior dislocation of the shoulder: long-term prognosis at the age of 40 years or younger. Knee Surg Sports Traumatol Arthrosc 2016; 24: 330-42

24. Simonet WT, Cofield RH. Prognosis in anterior shoulder dislocation. Am J Sports Med 1984; 12: 19-24.

25. Bishop JA, Crall TS, Kocher MS. Operative versus non-operative treatment after primary traumatic anterior glenohumeral dislocation: expected-value decision analysis. J Shoulder Elbow Surg 2011; 20: 1087-94.

26. Polyzois I, Dattani R, Gupta R, Levy O, Narvani AA. Traumatic First Time Shoulder Dislocation: Surgery vs Non-Operative Treatment. Arch Bone Jt Surg 2016; 4: 104-8.

27. Hovelius L, BG Augustini, H Fredin, O Johansson, R Norlin, J Thorling. Primary anterior dislocation of the shoulder in young patients. A ten-year prospective study. J Bone Joint Surg Am 1996; 78: 1677-84.

28. Sachs RA, Lin D, Stone ML, Paxton E, Kuney M. Can the need for future surgery for acute traumatic anterior shoulder dislocation be predicted? J Bone Joint Surg Am 2007; 89: 1665-74.

29. Olds M, Ellis R, Donaldson K, Parmar P, Kersten P. Risk factors which predispose first-time traumatic anterior shoulder dislocations to recurrent instability in adults: a systematic review and meta-analysis. Br J Sports Med 2015; 49: 913-22.

30. Henry JH, Genung JA. Natural history of glenohumeral dislocationd revisited. Am J Sports Med 1982; 10: 135-7.

31. Safran O, Milgrom C, Radeva-Petrova DR, Jaber S, Finestone A. Accuracy of the anterior apprehension test as a predictor of risk for redislocation after a first traumatic shoulder dislocation. Am J Sports Med 2010; 38: 972-5.

32. Salomonsson B, von Heine A, Dahlborn M, et al. Bony Bankart is a positive predictive factor after primary shoulder dislocation. Knee Surg Sports Traumatol Arthrosc 2010; 18: 1425-31.

33. Hoelen MA, Burgers AMJ, Rozing PM. Prognosis of primary anterior shoulder dislocation in young adults. Arch Orthop Trauma Surg 1990; 110: 51-4.

34. Hovelius L, Lind B, Thorling J. Primary dislocation of the shoulder: factors affecting the two year prognosis. Clin Orthop 1983; 176: 181-5.

35. Hovelius L. Anterior dislocation of the shoulder in teenagers and young adults: five-year prognosis. J Bone Joint Surg [Am] 1987; 69-A: 393-9. 